\title{
Genome-wide Analysis of R2R3-MYB Transcription Factors in Boehmeria nivea (L.) Gaudich Revealed Potential Cadmium Tolerance and Anthocyanin Biosynthesis Genes
}

\section{Xinkang Feng}

Institute of Bast Fiber Crops, Chinese Academy of Agricultural Sciences, Changsha 410205

Aminu Shehu Abubakar

Institute of Bast Fiber Crops, Chinese Academy of Agricultural Sciences, Changsha 410205

Kunmei Chen

Institute of Bast Fiber Crops, Chinese Academy of Agricultural Sciences, Changsha 410205

Chunming Yu

Institute of Bast Fiber Crops, Chinese Academy of Agricultural Sciences, Changsha 410205

Aiguo Zhu

Institute of Bast Fiber Crops, Chinese Academy of Agricultural Sciences, Changsha 410205

Jikang Chen

Institute of Bast Fiber Crops, Chinese Academy of Agricultural Sciences, Changsha 410205

Gang Gao

Institute of Bast Fiber Crops, Chinese Academy of Agricultural Sciences, Changsha 410205

Xiaofei Wang

Institute of Bast Fiber Crops, Chinese Academy of Agricultural Sciences, Changsha 410205

Pan Mou

Institute of Bast Fiber Crops, Chinese Academy of Agricultural Sciences, Changsha 410205

Ping Chen ( $\nabla$ chenping02@caas.cn )

Institute of Bast Fiber Crops, Chinese Academy of Agricultural Sciences, Changsha 410205

\section{Research Article}

Keywords: Ramie, MYB transcription factor, Expressed profiles, Anthocyanin biosynthesis, Cadmium stress, Protein interaction

Posted Date: February 24th, 2022

DOI: https://doi.org/10.21203/rs.3.rs-871051/v2 
License: (c) (i) This work is licensed under a Creative Commons Attribution 4.0 International License. Read Full License 


\section{Abstract}

MYB transcription factor (TFs) are one of the largest families in plants, and involved in many biological processes, including growth and development, metabolism and stress response. The MYB TFs has been extensively studied in various plant species. However, comprehensive information on MYB TFs in fiber crop ramie are still unknown. In the current study, a total of 105 BnGR2R3-MYB genes were identified from ramie genome and subsequently grouped into 35 subfamilies according to phylogeny divergence and sequences similarity. Chromosomal localization, gene structure, synteny analysis, gene duplication, promoter analysis, molecular characteristics and subcellular localization were accomplished using several bioinformatics tools. Collinearity analysis showed that the segmental and tandem duplication events is the dominant form of the gene family expansion, and duplications prominent in distal telomeric regions. Highest syntenic relationship was obtained between BnGR2R3-MYB genes and that of Apocynum venetum (88). Furthermore, transcriptomic data and phylogenetic analysis revealed that $B n G M Y B 60, B n G M Y B 79 / 80$ and BnGMYB70 might inhibit the biosynthesis of anthocyanins, and UPLCQTOF-MS data further supported the results. The quantitative real-time qRT-PCR and phylogenetic analysis indicated that the six genes (BnGMYB9, BnGMYB10, BnGMYB12, BnGMYB28, BnGMYB41, $B n G M Y B 78$ ) were cadmium stress responsive genes. Especially, the expression of $B n G M Y B 10 / 12 / 41$ in roots, stems and leaves all increased more than 10-fold after cadmium stress, and in addition they may interact with key genes regulating flavonoid biosynthesis. Thus, a potential link between cadmium stress response and flavonoid synthesis was identified through protein interaction network analysis. In conclusion, the studies increase our understanding of the functional roles of MYBs and provided valuable foundation for study of structural and regulatory genes in ramie.

\section{Background}

Transcription factors (TFs) are DNA-binding proteins that interact with cis-acting elements in the eukaryotic genes to regulate by either activation or inhibition of the transcription genes. The TFs are generally made up of four functional domains regions that include DNA binding domain, transcription regulation domain, oligomerization site, as well as the nuclear localization signal on the basis of which and number of residues in the domain TFs were classified[1]. MYB TFs, which are distinguished by the highly conserved MYB domain composed of one to four adjacent imperfect tandem repeats at the Nterminus, comprise one of the largest TF families in the plant kingdom[2]. Each repeat is approximately 50-53 amino acid residues in length, containing three a-helices that together form a helix-turn-helix $(\mathrm{HTH})$ secondary structure that interacts with the major DNA at the specific recognition site C/TAACG/TG during transcription[3]. On the contrary, the $\mathrm{C}$-terminal region is highly variable that leads to the wide range of regulatory roles of the MYB gene family[4]. According to the number of the adjacent MYB repeats, MYB genes are distinguished into the following four subfamilies: 1R-MYB, R2R3-MYB, R1R2R3MYB and 4R-MYB. Generally, the R2R3-MYB members, which have diverse functions, are the most extensively studied[5]. 
The MYB TF is present in all eukaryotes and was first reported in 1987 in Zea mays which was functionally characterized to partake in regulating anthocyanin biosynthesis[6]. Nowadays, with the advance of plant genome-wide association and molecular biology methods, an increasing number of studies have shown MYB proteins to play significant role in regulating plant primary and secondary metabolism, plant development, and in the response to various biotic and abiotic stresses[7-10]. CsTSI from Camellia sinensis for example, is reported to regulate biosynthesis of theanine in different tissues in tea plants[11]. While CaMYB31 is reported to involved in regulation of biosynthesis of capsaicinoids in Capsicum annum[12], DkMyb4 regulated proanthocyanidin biosynthesis in persimmon[13]. A number of MYBs linked to anthocyanin biosynthesis were identified in many species, such Arabidopsis (AtMYB75, AtMYB90, AtMYB113 and AtMYB114)[14-16], citrus (CsRuby 1 and CsMYB3)[17] and tomato (SIMYB12) [18] whereas others involved in cellular morphogenesis, secondary cell wall biosynthesis, meristem formation and cell cycle regulation[19-22]. A rice R2R3-MYB gene (OsCSA) directly triggers expression of sugar partitioning and metabolic genes during pollen and seed development[23]. GbMYB5 confers drought tolerance in cotton and transgenic tobacco plants[24]. Another rice MYB (OsMYB3R-2) exhibited enhanced cold tolerance by alteration in cell cycle and ectopic expression of stress genes[25].

Ramie is an important fiber crop in the Urticaceaes family which is also widely used as feed and other industrial raw materials[26-28]. The completion of the genome sequencing project of ramie [26, 29] provides a valuable data and platform for studying R2R3-MYB gene family and exploring its evolutionary characteristics. Despite the important role in plant specific processes, very limited reports are present to date on the functional characteristics of R2R3-MYB transcription factor in ramie. Therefore, this study comprehensively investigated and reported gene structure, gene duplication, chromosomal localization, phylogeny and cis-acting elements of the BnGR2R3-MYB gene. Transcriptome expression profiling coupled with UPLC-QTOF-MS was used to identify BnGMYBs involved in anthocyanin synthesis. Some selected MYBs were induced under cadmium stress and their expression explored to provide an idea and direction into their function in the ramie. In addition, protein interaction network analysis found a potential link between cadmium stress and flavonoid synthesis.

\section{Results And Discussion}

\section{Identification and sequence feature of ramie MYB genes}

To identify the MYB family genes in ramie genome, the amino acid sequence of hidden Markov model (HMM) profile of the Pfam MYB domain (PF00249) was used as a query and obtained 245 amino acid sequences that contain MYB or MYB-like repeats. Subsequent processing with FGENESH-M returned 211 sequences. After confirmation of the presence of MYB domains base on the PROSITE, Pfam and NCBICDD analyses, we finally established a total of 200 non-redundant MYB proteins which included 89 MYBrelated proteins (1R-MYB), 105 R2R3-MYB (2R-MYB), 5 R1R2R3-MYB (3R-MYB) and 1 4R-MYB proteins. According to previous reports, R2R3-MYB family members not only represent more than half of the proportion of the total MYB proteins, but also contain most functional genes[3]. Therefore, we selected only the R2R3-MYB for further study. Based on their order on the chromosome, the R2R3-MYB genes were 
renamed BnGMYB1 to BnGMYB102. Three of the 105 R2R3-MYBs (Maker00022368, Maker00031449, and Maker00022368) which could not be conclusively mapped to any chromosomes, were renamed BnGMYB103 - BnGMYB105 respectively (Table S1). The molecular weight of the protein as obtained using ProtParam analytical tool revealed BnGMYB52 as the smallest with 135 amino acids (aa), and $B n G M Y B 1$ (593 aa) the largest. The MW of the proteins ranged from 15.6 to $65.0 \mathrm{kDa}$, and the pl ranged from 4.56 ( $B n G M Y B 23$ ) to 10.08 ( $B n G M Y B 96)$. All the R2R3 but two are localized in the nuclear region, whereas the remaining two were in either chloroplast or cytoplasm (Table S1).

R2, R3 repeats are significantly conserved sequences within the MYB domain regions. Sequence logos were constructed to investigate the homologous domain features and the frequency of amino acids (Fig. 1). There were 108 amino acids conserved residues in the BnGR2R3-MYB. 11 residues in the R2 and 6 in the R3 were completely among all BnGMYB. These included the most prominent series of evenly distributed and highly conserved triplet tryptophan (Trp., W) residues in each repeat at positions 9, 29 and 49 of the R2 repeat; 28 and 47 of the R3 repeat. These characteristic amino acids are known to play an important role in sequence-specific DNA binding, and are considered landmarks for plant MYB proteins [30]. R3 repeats in BnGMYB66, BnGMYB56, BnGMYB67 have the last Trp residue replaced with Tyrosine (Tyr., Y) or phenylalanine (Phe., F) which were consistent with those of other species[31, 32]. Insertion of leucine residue (Leu., L) between second and third helices of the $\mathrm{R} 2$ repeat have been reported to represents evolutionary relationship of R2R3-MYB domain[33]. In this study, 83 (79.05\%) BnGR2R3-MYBs were observed to have Leu-38 inserted between the two helices (Table S2).

Fig. 1. Consensus sequence and the level of conservation of R2R3-MYB domains from ramie. The sequence logos of the R2 and R3 MYB repeats are based on full-length alignments of all ramie R2R3MYB domains. The bit score exhibits the information content for each position in the sequence. The position of the three a-helices were marked (Helix 1 to 3 ). The conserved tryptophan residues (Trp, W) in the MYB domain are marked with black asterisks.

\section{Gene structure and motif composition of BnGMYBs}

We performed a phylogenetic analysis of R2R3-MYB proteins using neighbor-joining [34] and maximum likelihood (ML) methods with 1000 bootstrap replicates. The tree topologies obtained using the two methods were largely similar with a very few variations in the gene classification (Fig. 2a and Figure S1). Since the ML method can choose the optimal alternative model and optimize the evolutionary tree with certain topological structure and branch length, we adopted it for further characterization. The R2R3-MYB members of ramie were subdivided into 30 subgroups (designated A1-A30 in this study) using Arabidopsis MYB proteins as reference [3]. Nine clades only contained ramie R2R3-MYB members, and four genes did not fit into any subgroup. 
To investigate the relationship between gene structural function and evolution, we analyzed the structural diversity of the BnGR2R3-MYB family, which showed genes having the same genetic structure clustering together as obtained in the phylogenetic tree (Fig. 2a). MYB conservative motifs as obtained using MEME online tool (Fig. 2b) revealed the presence of 20 conserved motifs in the ramie MYB proteins (Table S3). The gene structure analysis showed most of gene coding sequences disrupted by introns, except for almost all members from A30 subgroup, ungrouped BnGMYB27/37 and BnGMYB72 (Fig. 2c). The number of introns ranges from zero to eleven, with most genes having three exons and two introns in line with previous reports[35]. Interestingly, the majority of intron insertions occur in R2, R3 conserved domains indicating the important role of these conserved domains in plants. The highly conserved gene structure and domain influence each other, which provided important evidence for the division of subgroups. Similarly, protein architecture was conserved within a specific subgroup. Phylogenetic tree, gene structure, and motif analysis suggested similar functions for MYB proteins within the same subgroup. In addition to the conserved motif representing R2R3, other similar motifs were shared by the BnGR2R3-MYB members within the same subgroup, for example, motifs 11,17 , and 19 were unique to members in clades $A 25, A 23$ and $A 1$ respectively.

Fig. 2. Phylogenetic relationships, gene structure and architecture of conserved protein motifs in BnGR2R3-MYB genes. A. The maximum likelihood phylogenetic includes 105 R2R3-MYB proteins from ramie and 23 representatives from Arabidopsis B. The motif architecture of ramie MYB proteins. 20 different motifs are displayed in different colored boxes. C. Exon-intron structure of ramie MYB genes. Green boxes indicate exons; blue boxes indicate untranslated 5'- and 3'- regions; black lines indicate introns. R2, R3 MYB domain are highlighted by boxes of different shape and color.

\section{Chromosomal distribution and synteny analysis of BnGMYB genes}

Ramie R2R3-MYB genes were unevenly distributed in the species 14 chromosomes (Fig. 3), with Chr10 having the largest number (14) followed by Chr4 (13) and the least number (2) found on Chr12. No significant correlation was obtained between the chromosome length and the number, while some of the genes were concentrated at either top or bottom of the chromosome. The closer the genes are to each end, the more likely they are to crossover[36].

Based on BLASTP and MCScanX methods, we investigated the gene duplication events. Reference to Holub[37] for a description of tandem duplication, among the BnGR2R3-MYB genes, the blue lines in Fig. 3 showed four pairs of tandemly duplicated genes (BnGMYB32/33,

BnGMYB51/52, BnGMYB61/62, BnGMYB93/94) located in Chr5, Chr7, Chr8, Chr13, respectively, whereas the red lines showed 39 segmental (37.1\%) duplication pairs between BnGR2R3-MYB genes (Table S4) including two MYB-related genes (Maker00025472; Maker00000772) and one 3R-MYB gene (Maker00072694). Some BnGMYBs located on Chr13, Chr6 and Chr4, such as BnGMYB91, BnGMYB90, 
BnGMYB89, BnGMYB21, BnGMYB44 and BnGMYB45, were involved in multiple duplication events. BnGMYB89 (Chr12) was however interrelated with BnGMYB90/91 (Chr13) and $B n G M Y B 45$ (Chr6). Interestingly, all segmentally duplicated genes fall into A30 subgroups together with A. thaliana AtMYB45 (Fig. 2a) making us speculate that this specific population of R2R3-MYB genes duplication in ramie might be closely related to photomorphogenesis[38], and might play a vital role in the growth and development of the plant. The segmental and tandem duplication events are major derivers of the gene family expansion[39].The positive pressure criteria were selected based on M. Lynch[40]: $\mathrm{Ka} / \mathrm{Ks}<1$ stands for purifying selection, $\mathrm{Ka} / \mathrm{Ks}=1$ means neutral selection, while $\mathrm{Ka} / \mathrm{Ks}>1$ signifies positive selection. We found a high sequence divergence value among the four segmental gene pairs. These sequences diverged greatly and evolution distance was long, owing to the occurrence of a large number of synonymous mutations. The remaining segmental and tandem duplicated BnGMYB gene pairs were $\mathrm{Ka} / \mathrm{Ks}<1$ except $B n G M Y B 66 / 67(\mathrm{Ka} / \mathrm{Ks}=1.04)$. These results suggest that the ramie R2R3BnGMYB gene family had evolved under the effect of purifying selection (Table S4).

Fig. 3. Schematic representations for the chromosomal distribution and inter-chromosomal relationships of ramie R2R3-MYB genes. Gray lines indicate all synteny blocks in the ramie genome, and the red lines indicate duplicated MYB gene pairs. Gene and chromosome names are displayed on the tip and inside of the chromosome, respectively.

Syntenic maps of ramie with five other plants, comprising dicots ( $A$. thaliana, $C$. sativa, $A$. venetum) and monocots (rice and maize) conducted to further explore potential evolutionary mechanisms of BnGR2R3MYB genes, revealed $88,58,53,8$ and 4 numbers of orthologous gene pairs (Fig 4). Collinearity analysis showed highest collinearity segments of the ramie with $A$. venetum and $C$. sativa in consistent with the phylogenetic divergence time estimation which supported the placement of ramie within the same clade as $C$. sativa[29]. Some of the BnGMYBs were found to be associated with multiple syntenic gene pairs, particularly BnGMYB89 and four $A$. thaliana genes, suggestion that these genes might have played an important role in MYB gene family during evolution. Remarkably, the number of collinear gene pair between ramie and $A$. venetum, $C$. sativa, and $A$. thaliana (dicotyledonous) was far greater than between ramie and $O$. sativa/Z. mays (monocotyledonous) [29, 41, 42]. 19 BnGMYBs identified in dicotyledonous plant were absent in monocotyledonous plant, suggestion that these orthologous pairs (19 BnGMYBs) formed after the divergence of dicotyledonous and monocotyledonous plants. Additionally, one collinear pair (BnGMYB64) was identified in all the six detected plants, indicating that these orthologous pairs might had already existed before the ancestral divergence (Table S5). 
Fig. 4. Synteny analyses of MYB genes between ramie and five representative plants: $B$. nivea, $C$. sativa, $A$. venetum, $O$. sativa, $Z$. mays and $A$. thaliana. Gray lines in the background indicate the collinear blocks within ramie and other plant genomes, while the red lines highlight the syntenic MYB gene pairs.

\section{Comparative phylogenetic analysis of BnGMYB and R2R3-MYB family from six different plant species}

A Neighbor-joining [34] phylogenetic tree was constructed using the 105 full-length R2R3-MYB protein sequences of ramie and those from Arabidopsis (126), rice (81), maize (156), tomato (121) and pineapple (93) and the result shown in Fig. 5 and S2. The phylogenetic analysis generated 35 clades (C1-C35) among six species and the numbers of the MYB members in each species were listed (Fig. 5). The fact that this tree was in good agreement with the classification results of $A$. thaliana[3] and other plants[31, 32 , 43] demonstrated the reliability of the data. Moreover, the comprehensive phylogenetic analysis of the R2R3-MYB proteins in these six species might provide more evolutionary historical clues. For example, monocotyledon MYB proteins in the same group were often found clustered together, while MYB proteins from dicotyledonous plants were clustered side by side in lateral branches. Meanwhile, several clades were found only in some particular species. For example, none of rice, maize, or pineapple MYB members fall in the $\mathrm{C} 5, \mathrm{C} 11$, and $\mathrm{C} 16$. Similarly, C31 had only monocotyledons. This indicated a great evolutionary distance between monocotyledons and dicotyledons, and that these specific MYB genes might have evolved after divergence of the two classes. Apart from these, all other clades contained at least a member from each of the six species, suggesting common ancestry before the species diverged. The varying number of MYBs from the species in a particular clade is an indicative that MYB genes expansion may be more active in some plant species, and this expansion inequality may be related to the well conserved chromosome karyotype [44]. Furthermore, MYBs in the same clade may have similar function with to the model Arabidopsis, thus provide direction for functional identification of ramie MYB proteins $[3,38,45]$.reference Polyploidy and subsequent genes loss exist in most species is an important driving force of species evolution. Based on the comparative genomic analysis, it could be inferred (Figure S3) that ramie has undergone at least one round of genome-wide duplication events. Phylogenetic analysis of MYB gene family showed that the number of BnGMYB members in some clades was consisted with rice and maize. Ramie also undergone the genome-wide duplication event common to rice[46] and maize[47]. 
Fig. 5. Neighbor-joining [34] tree showing relationships among R2R3-MYB proteins from $B$. nivea [48], $A$. thaliana (At), O. sativa (Os), Z. mays [49], S. lycopersicum (SI) and A. comosus (Ac). MYB proteins from the six species were designated as $\mathrm{C} 1$ to $\mathrm{C} 35$. The table on the right indicated the number of the subgroup members and function of clades in each species.

\section{Analysis of the cis-acting elements of the BnGR2R3-MYB genes and Gene ontology annotation}

The upstream promoter regions (1000bp) of the BnGR2R3-MYB genes were predicted for better understanding of the R2R3-MYB genes functions. There were total of 41 responsive cis-acting elements (Fig. 6 and Table S6), which made up of 22 light responsive elements, 8 phytohormone responses, 6 plant growth development-related responses and 5 abiotic stresses (adversity). Light response elements being the most abundant are present in all genes. Box-4, G-box (light responsiveness), ARE (anaerobic induction) and ABRE (abscisic acid responsiveness) were the most prominent elements, and presence in more than 100 of the 105 BnGR2R3-MYB genes, suggesting that these elements might play an important role in regulating gene expression. A total of $65(61.9 \%)$ genes were found to contain ABRE. Genes containing anaerobic induction, wound response, low temperature response, and drought stress related elements accounted for $55.2 \%, 26.7 \%, 24.8 \%$ and $17.1 \%$, respectively. In addition, there are 118 elements in all genes involved in the MeJA response. These results indicate the potential function of BnGR2R3MYB genes in response to various stresses.

Almost all BnGR2R3-MYB genes as obtained in this study contained several cis-acting elements associated with abiotic stresses such as anaerobic induction, drought, low temperature, defense and stress, which is in line with literature[50]. The abiotic stress responsive elements were mostly clustered together, for example, A29 and A30 phylogenetic subgroups in Figure 6 (corresponding to the C32 and C33 subgroups in Fig. 5) which were consistent with their functional clustering and might be related to the function of gene groups. This further reflected the accuracy of the results.

Fig. 6. Analysis of cis-elements from promoter region of the BnGR2R3-MYB genes. The figure on the left shows the distribution of four cis-acting elements upstream promoter regions (1000bp). On the right is the heat map of the quantity of plant growth, stress responsive and phytohormone responsive ciselements, represented in brown, blue and green, respectively, circle sizes indicate the number of ciselements. 
Gene ontology (GO)[51] annotations of 105 BnGMYB proteins is displayed in Figure 7 and indicate the proteins involvement in biological process (BP), cellular component (CC) and molecular function (MF). The GO term "binding" (GO: 0005488) best described the greatest number of genes (96, 92.38\%), and "single-organism developmental process" (GO: 0044767), "developmental process" (G0:0032502) were significantly enriched in top 20 of biological process. These GO annotations of BnGMYB proteins were in agreement with the experimental findings in other species[52-54].

Fig. 7. BnGMYB Gene Ontology (GO) functional classification. (A) BnGMYB TFs were divided into categories with three independent ontologies. (B) The Top 20 of GO enrichment in biological processes.

\section{Expression profiling of BnGR2R3-MYB genes with RNA-seq in different tissues}

We analyzed transcript levels obtained from transcriptome data of 9 different samples comprising tissues (phloem, root, leaf) and developmental stages of ramie to profile expression of BnGR2R3-MYB. The results (Fig. 8 and Table S7a) showed most BnGMYBs to have exhibited different expression patterns in line with what was obtained in other plants [55]. Three BnGMYBs (BnGMYB15; BnGMYB75; BnGMYB101) were not detected in all nine samples, suggesting that they might be pseudogenes, or only expressed in other tissues. Hierarchical cluster analysis of the BnGMYBs from 9 the different samples enabled grouping them into three: high expression, preferential expression, and relatively lower expression. The expression grouping in most cases indicated that the expression patterns of genes were significantly different. However, some closely related MYB genes showed highly similar transcript levels, for example, three members of $A 30$ (BnGMYB89/90/91) all belonged to high expression group. A total of 57 genes were expressed in all tissues, and 16 from which showed constitutive expression (FPKM $>2$ ). In order to better understand the preferential expression of genes, we filtered genes based on meeting two set rules: FPKM>2 (at least in one tissue); tissues with the highest expression levels should have twice as much expression in at least one of the other tissues. Based on this, 11 genes in phloem_third period, 19 genes in leaf of variety ZZ_1, 22 genes in terrestrial root were found to have exhibited preferential expression over the others (Table S7c). 
Fig. 8. Hierarchical clustering of expression profiles of ramie R2R3-MYB genes in 9 samples including different tissues and developmental stages. Log2 (FPKM +1) values were displayed according to the color code. Detailed FPKM values were listed in Table S7.

In our previous study, we conducted transcriptomic and non-targeted metabolomic studies of two ramie varieties which have distinct differences in leaf color, HX_1 (red-leaf variety) and ZZ_1 (green-leaf variety), and revealed that the color differences between the duo was due to differences in expression of anthocyanin synthesis pathway related gene, with no clarity in the absolute content of ramie anthocyanin and the function of regulatory gene (e.g., transcription factors)[56]. In this study, we examined the composition and content of anthocyanin in the two varieties. The results showed that the anthocyanin in ramie was mainly Cyanidin-3-rhamnoside (Fig. 9). The Cyanidin-3-rhamnoside content in HX_1 was as computed based on the peak value was $3.147 \mu \mathrm{g} / \mathrm{ml}$., whereas the anthocyanin was almost absent in ZZ_1. Thus, the differences in the leaf color were attributed to this.

Fig. 9. UPLC-QTOF-MS method for the detection of anthocyanin in HX_1 and ZZ_1.

Several transcription factors including MYB have been reported to interact with key genes involved in anthocyanin synthesis [57]. A total of 14 differentially expressed MYB transcription factors were searched in the transcriptome data, of which five were up-regulated and nine were down-regulated, with the highest differential expression ploidy reaching 17-fold, implying that MYB was very active in two varieties of different colors (Table S7b). Therefore, we selected these MYBs for the construction of phylogenetic tree with 50 anthocyanin synthesis-related MYB genes identified in other plants and R2R3-MYBs of the model plant Arabidopsis. As shown in Figure 10, the 50 anthocyanin synthesis-related MYBs were grouped into five subgroups, and three of the 14 differentially expressed MYB genes were clustered into these subgroups: $B n G M Y B 60$ (B2), BnGMYB79/80 (B3), and $B n G M Y B 70$ (B4). All three genes which are homolog of PtrMYB182[58]/VvMYBC2L3[59], AtMYB123 (TT2)[60], and AtMYB82[61] involve anthocyanin synthesis repression were down-regulated in HX_1 (Figure 10). No ramie MYB was found in the B1 subfamily that positively regulates anthocyanin, and therefore anthocyanin synthesis in ramie is not regulated by the B1 subgroup MYB transcription factors. It will be envisaged that, up-regulation of $B n G M Y B 60$ (B2), BnGMYB79/80 (B3), and BnGMYB70 (B4) caused the inhibition of ZZ_1 anthocyanin synthesis, which ultimately affected color formation. 
Fig. 10. Phylogenetic tree and gene expression analysis of R2R3-MYB transcription factors associated with anthocyanin synthesis.

\section{Expression profiles of BnGMYBs under $\mathrm{Cd}^{+2}$ stress}

Ramie is a promising plant for remediation of heavy metal contaminated land and has high cadmium tolerance and accumulation capacity[62]. An increasing number of studies have linked R2R3-MYB genes to various stress responses and regulation. However, there was not much information on the involvement of MYB genes in cadmium tolerance response in ramie. We thus screened 12 MYBs candidates by combining RNA-seq expression profiles, homologous gene relationships, as well as their expression patterns under cadmium stress.

We analyzed them in three dimensions: treatment time, species and tissue site. The results (Fig. 11) showed varied expression patterns of the BnGMYB genes under different $\mathrm{Cd}^{2+}$ treatment times with four different trends of continuously rising, rise and fall, continuously falling, and rising after falling. Majority of the genes showed characteristics fall upon treatment before rising. The expression tendency of 11 genes were consistent in the two ramie cultivars, while some genes responded more strongly in HX_1 than in ZZ_1, such as BnGMYB28, BnGMYB9, BnGMYB78, indicating that the response mechanisms to $\mathrm{Cd}^{2+}$ stress is similar in the two cultivars. The degree of response in different parts of the plant under the same stress was also different, however, all the genes were up-regulated in the leaves on cadmium treatment. Combining the results of different treatment times and tissue sites, we found that genes such as BnGMYB9/11/41/89 showed a bottom-up response pattern in the order (root-stem-leaf) which may relate to cadmium transport and stress signaling.

The expression of BnGMYB10/12/41 in roots, stems and leaves all increased more than 10-fold after cadmium stress, and phylogenetic analysis placed these three genes into the flavonoid synthesis MYB subgroup (C1), suggesting a potential association between flavonoid synthesis and cadmium tolerance[3]. For further understanding the association of flavonoid synthesis and cadmium stress, we selected AtMYB4[63], AtMYB30[64], AtMYB49[65], and AtMYB96[66] which are respectively homologs of $B n G M Y B 41, B n G M Y B 28, B n G M Y B 9, B n G M Y B 78$ with established role in in defense responses, and AtMYB4[63], AtMYB12[67], TT2[68] (homologs of BnGMYB10/ BnGMYB12/ BnGMYB41, respectively) involved in regulating flavonoid synthesis pathway in Arabidopsis thaliana for protein interaction network analysis (Fig. 12). These MYB TFs were found to interact with flavonoid biosynthetic genes, in addition to being tightly linked to some resistance proteins. BnGMYB12 and BnGMYB41 interacted with LPP2 (lipid 
phosphatase 2)[49], SAD2[69] (sensitive protein to $A B A$ and drought 2), respectively. A number of proteins in the flavonoid metabolic pathway have been reported to interact with jasmonic acid-induced oxygenase 4 (JOX4)[70]. We also found that BnGMYB9/28/78 interacts with many important stress resistance proteins such as $S I Z 1$ [71], $R D 22[72], Y A K 1[73], A B I 5[72]$, etc. Interestingly, we found an interface between the flavonoid metabolic pathway and cadmium stress, the TGG1 (thioglucoside glucohydrolase 1) node connecting two possible stress coping mechanisms. This finding provides a basis for understanding the association of flavonoid synthesis and stress regulation in ramie.

Fig. 11. The relative expression levels of selected MYB genes in two ramie varieties in different tissue sites (root, stem, leaf) at different periods under $\mathrm{Cd}^{+2}$ treatment (0h, $\left.6 \mathrm{~h}, 12 \mathrm{~h}, 24 \mathrm{~h}, 48 \mathrm{~h}\right)$. A represents root; $B$ represents stem; $C$ represents leaf. The error bar represents the standard deviation of the three biological duplicates. * represents significant difference $(P<0.05)$, ** represents extremely significant difference $(P<0.01)$.

Fig. 12. The protein-protein interaction network for BnGR2R3-MYB proteins based on their Arabidopsis orthologs. The red solid circles indicated cadmium stress-related MYB transcription factors, the olive squares represented stress-response proteins, and the green squares were flavonoid synthesis related proteins.

\section{Conclusions}

A total of 105 BnGR2R3-MYBs unevenly distributed among 14 chromosomes, phylogenetically divided into 35 distinct subfamilies, were identified in ramie. The segmental duplication events especially prominent in distal telomeric regions, played a crucial role in the expansion of BnGR2R3-MYB gene family. The result showed highest orthologous gene pairs between ramie and Apocynum venetum (58) than other plants including Arabidopsis. Expression analysis led to the identification of tissue preferential and cadmium stress responsive expression patterns of the BnGR2R3-MYB genes. Additionally, putative functions of ramie MYB genes were assigned based on the phylogenomic results and gene expression data. We performed comparative transcriptome analysis between HX_1 and ZZ_1 coupled with phylogenetic and UPLC-QTOF-MS analysis and identified BnGMYB60, BnGMYB79/80 and BnGMYB70 as potential anthocyanin biosynthesis repressor genes. Expression analysis of selected BnGMYB under cadmium stress resulted in the identification of $B n G M Y B 9 / 10 / 12 / 28 / 41 / 78$ as potential stress regulators and three MYBs (BnGMYB10/12/41) in addition, flavonoid biosynthesis regulatory genes which is consistent with their annotated homologues in Arabidopsis. Thus, this work provided valuable 
information on BnGR2R3-MYB gene family with respect to Cadmium stress tolerance and anthocyanin biosynthesis.

\section{Materials And Methods}

\section{Identification of MYB protein in Boehmeria nivea (L.) Gaudich}

For the comprehensive identification of the ramie MYB TFs, 168 MYB family sequences and 97 MYBrelated family sequences ( $A$. thaliana), 208 MYB family sequences and 85 MYB-related family sequences (G. arboreum), 130 MYB family sequences and 106 MYB-related family sequences (O. sativa) were retrieved from the PlantTFDB (http://planttfdb.gao-lab.org/). The protein sequences of these species were then used as reference to filter the possible sequence of target specie by Blast Wrapper, with expectation cut-off value (E-value) of e-5 set as threshold significance. The sequences obtained were aligned to Swissport Database / Reference Species Whole Protein Sequence Library, and screened by annotation information. The resulting datasets of MYB sequences were confirmed based on the completeness of MYB domains using Pfam, CDD and SMART with an E-value set at 0.01. To correct for deletion of some conserved sites, FGENESH-M (http://www.softberry.com/berry.phtml) was used to predict multiple variants potential genes in genomic DNA. All the resulting ramie R2R3-MYB proteins were manually inspected to ensure that it contained two complete MYB domains and ultimately identified and classified as members of the MYB family in Boehmeria nivea. Length of sequences, molecular weights, GRAVY and pl of the MYB proteins were obtained using the ExPASy online tool[74]and subcellular localization predicted using the pLoc-mPlant tools for Batch Prediction.

\section{Sequence analysis and structural characterization of BnGR2R3-MYB genes}

Multiple sequence alignments of the MYB domains sequences were performed using MEGA-X with default parameters. The deduced amino acid sequences of the MYB motifs were adjusted manually in Jalview software and WEBLOGO used to show up the sequence logos of R2 and R3 MYB domain repeats. The exon-intron organizations of the BnGR2R3-MYB genes, including intron distribution patterns, phases, intro-exon boundaries and highlighted region of the MYB domains were graphically displayed with the aid of TBtools[75]. The Conserved motifs of the ramie MYB proteins were predicted by using the MEME[76] version 5.1.0 with optimized parameters: zero or one per sequence; maximum number of motifs set at 20, and visualized in TBtools.

\section{Analyses of chromosome distribution, gene duplication and synteny for BnGR2R3-MYB genes}

The detailed chromosome distribution information of each BnGR2R3-MYB genes was obtained from the ramie genome annotation documents. Circos[77] was used to locate all the BnGR2R3-MYB genes on the ramie chromosome. Tandem, segmental duplication and collinearity within species were obtained by using Multiple Collinearity Scantoolkit (MCscanX)[78]. Synteny blocks of the orthologous R2R3-MYB genes between ramie and other species were obtained also using MCscanX and both results visualized in 
TBtools[75]. Non-synonymous and synonymous substitution of each duplicated BnGMYB genes were calculated using KaKs_Calculator 2.0[79].

\section{Phylogenetic analysis and classification of ramie BnGR2R3-MYB proteins and WGDI analysis}

Multiple sequence alignments of the R2R3-MYB proteins from ramie and Arabidopsis thaliana were performed using MEGA 7.0 with default parameters[80] and neighborhood linkage [34] phylogenetic tree constructed with the following parameters: Poisson model; pairwise deletion; and 1000 bootstrap replications. R2R3-MYB proteins from other species (Arabidopsis[37], rice[54], maize[81], tomato[32], pineapple[43]) were obtained based on the description in corresponding literatures, and adopted same.

To establish WGD events, paralogous genes of ramie were detected using all-vs-all homology searches in BLASTP with an E-value threshold of 1e-5. Syntenic blocks within a genome were identified based on the detected homologous gene pairs using MCscan.

\section{Identification of the cis-elements of BnGR2R3-MYB genes and GO annotation}

The promoter regions of the BnGR2R3-MYB genes, 1000 bp genomic DNA upstream sequences of each of the 105 BnGR2R3-MYB were selected and the cis-elements predicted using PlantCare (http://bioinformatics.psb.ugent.be/web tools/plantcare/html/). Response class elements such as lightresponsive, plant growth, stress-responsive and phytohormone-responsive were filtered and the cis-acting elements visualized in TBtools, and displayed with a heatmap. Following blasting in BLASTP against Swissport database, functional annotation of BnGMYB proteins was performed in Blast2GO Tool and subsequent mapping Gene Ontology (GO) terms, and visualization with R ggpolt2.

\section{Expression patterns of BnGR2R3-MYB genes in the representative tissues of the ramie}

A total of 9 transcriptome data samples comprising different tissues (phloem, root, leaf), different developmental stages and growing condition (hydroponics or terrestrial) obtained from Genome Sequence Archive database were used. Transcript abundance of the BnGR2R3-MYB genes was calculated as fragments per kilobase of exon model per million mapped reads (FPKM). Log2(FPKM +1 ) values were displayed according to the color code. Detailed FPKM values were listed in Table S7.

\section{Determination of anthocyanin content by UPLC-QTOF-MS}

Anthocyanin content was determined following the method of Wang et al[82]. $0.4 \mathrm{~g}$ of the leaf samples of each of HX_1 and ZZ_1 was extracted in a brown glass tube with $4 \mathrm{ml}$ of methanol/formic acid (9:1, v/v). Samples were extracted at $4{ }^{\circ} \mathrm{C}$ for $12 \mathrm{~h}$ followed by centrifugation at $3900 \mathrm{rpm}$ for $20 \mathrm{~min} .2 \mathrm{ml}$ of the supernatant was filtered into a new brown glass tube using a $0.22 \mu \mathrm{m}$ nylon membrane. A Waters ACQUITY UPLC I-Class-Xevo G2-XS QTOF/PDA eLambda Detector was used on an ACQUITY UPLC CSH C18 $(2.1 \mathrm{~mm} \times 100 \mathrm{~mm}$ i.d., $1.7 \mu \mathrm{m})$ column. Mobile phase A was acetonitrile with $5 \%$ formic acid and mobile phase $B$ was water with $5 \%$ formic acid. The photo-diode array (PDA) wavelength range was from $190 \mathrm{~nm}$ to $800 \mathrm{~nm}$. Peonidin-3-glucoside chloride $(15 \mu \mathrm{g})$ was added to the sample as a standard. The 
injection volume was $1 \mu \mathrm{l}$ for all samples and positive ion mode set for mass. The ESI source parameters were set according to the literature[82]. The MS/MS for fragments were over the range from $50 \mathrm{~m} / \mathrm{z}$ to $1000 \mathrm{~m} / \mathrm{z}$ in a continuum mode. The ramp range was from $6 \mathrm{~V}$ to $80 \mathrm{~V}$.

\section{Stress treatment $\left(\mathrm{Cd}^{2+}\right)$ of ramie under hydroponic conditions}

Two ramie varieties (ZZ_1; HX_1) were used for cadmium stress treatment. The two species have conspicuous leaf color attributed by different anthocyanin content[56] Shoots from each of the varieties at similar growth stage during the same period were selected for hydroponic cutting according to Gao et al., [83]. These were monitored for 15 days and seedlings with inconsistent growth were removed. $\mathrm{CdCl}_{2}$ to a final concentration of $50 \mathrm{mg} / \mathrm{L}$ was added and three separate biological replicates were sampled at $0,12,24$, and $48 \mathrm{~h}$ after the treatments. All samples were frozen quickly in liquid nitrogen, and stored at $-80^{\circ} \mathrm{C}$ until used.

\section{BnGMYB protein-protein interaction network}

Orthologous relationships of eight selected cadmium-responsive MYB regulatory genes were determined between Arabidopsis thaliana and ramie using OrthoVeen2[84]. Prediction of interactions between BnGMYB proteins and other proteins based on the Arabidopsis homologs was obtained using the online program STRING version 11.5 with high confidence $>0.700$ [85], filtered genes were used to construct the correlation network. The interaction network was visualized inCytoscape v3.8.2.

\section{RNA isolation and expression analysis of BnR2R3-GMYB genes}

Total RNA was extracted using EasySpin Plus plant RNA rapid extraction Kit (Aid-lab Biotechnologies Co., Ltd). The RNA was reverse-transcribed (Thermo Scientific RevertAid First cDNA Synthesis Kit) into cDNA and quantitative RT-PCR ( $\mathrm{qPCR}$ ) analysis conducted using gene specific primers (Table S8). 18s gene was used as internal control. The qPCR was conducted using SYBR Green Premix Pro Taq HS qPCR Kit (Accurate Biotechnology Co., Ltd) on a CFX96 Touch Deep Well Real-Time PCR System (Bio-Rad) according to standard procedure. Relative transcript levels were calculated using the $2^{-\Delta \Delta C t}$ formula and the result displayed using histograms drawn with GraphPad Prism 8 software and all the histograms merged using Adobe Photoshop (2020) software. All qPCR analyses were performed with three biological and technical replications.

\section{Abbreviations}

HTH: helix-turn-helix; MEME: Multiple Em for Motif Elicitation; NJ: Neighbor-Joining; ORF: open reading frame; GSDS: Gene structure display server; PlantTFDB: Plant Transcription Factor Database; RNA-seq: RNA-sequencing; SMART: Simple Modular Architecture Research Tool; TAIR: The Arabidopsis Information Resource; TFs: Transcription Factors; qPCR: Quantification real-time PCR; MWs: Molecular weights; MEME: Multiple Expectation maximization for Motif Elicitation; pls: Isoelectric points; GRAVY: Grand average of hydropathicit; HX_1: Hongxuan No.1 variety; ZZ_1: Zhongzhu No.1 variety 


\section{Declarations}

\section{Acknowledgements}

We would like to thanks the Institute of bast fiber crops, Chinese Academy of Agricultural Sciences for kindly supplying the experimental material.

\section{Author Contributions}

PC, XKF conceived and designed the experiments; XKF, AGZ, JKC, XFW, PM performed the experiments; $\mathrm{XKF}, \mathrm{CMY}, \mathrm{KMC}, \mathrm{GG}$, analyzed the data; XKF, ASA, PC wrote and reviewed the paper. All authors have read and approved the manuscript.

\section{Funding}

This work was supported by the Agricultural Science and Technology Innovation Project of the Chinese Academy of Agricultural Sciences (CAAS-ASTIP-2020), the China Agriculture Research System of MOF and MARA (CARS-16-E03).

\section{Availability of data and materials}

Raw reads, assembly genome sequences and corresponding annotation used in this study source cngb (China National GeneBank) database. Project: CNP0001467, review link: https://db.cngb.org/cnsa/project/CNP0001467/reviewlink. Bast fiber tissue transcriptomics in 5 developmental stages source cngb under project: CNP0001453, review link: https://db.cngb.org/cnsa/project/CNP0001453/reviewlink. We have received administrative permission to access and use these. The transcriptome of root and leaf used in this study are available through the NCBI SRA (PRJNA717370, GSE98903).

\section{Ethics approval and consent to participate}

The plant materials are owned by the Institute (the Institute of bast fiber crops, Chinese Academy of Agricultural Sciences) and we have the right to use them. Sampling of plant materials were performed in compliance with institutional, national, and international guidelines. The materials were publicly available for non-commercial purposes. No specific permits were required.

\section{Consent for publication}

Not applicable.

\section{Competing interests}

The authors declare no conflict of interest. 


\section{References}

1. Liu L, White MJ, MacRae TH: Transcription factors and their genes in higher plants functional domains, evolution and regulation. Eur J Biochem 1999, 262(2):247-257.

2. Riechmann JL, Ratcliffe OJ: A genomic perspective on plant transcription factors. Curr Opin Plant Biol 2000, 3(5):423-434.

3. Dubos C, Stracke R, Grotewold E, Weisshaar B, Martin C, Lepiniec L: MYB transcription factors in Arabidopsis. Trends Plant Sci 2010, 15(10):573-581.

4. Ogata K, Kanei-Ishii C, Sasaki M, Hatanaka H, Nagadoi A, Enari M, Nakamura H, Nishimura Y, Ishii $\mathrm{S}$, Sarai A: The cavity in the hydrophobic core of Myb DNA-binding domain is reserved for DNA recognition and trans-activation. Nat Struct Biol 1996, 3(2):178-187.

5. Jiang CK, Rao GY: Insights into the Diversification and Evolution of R2R3-MYB Transcription Factors in Plants. Plant Physiol 2020, 183(2):637-655.

6. Paz-Ares J, Ghosal D, Wienand U, Peterson PA, Saedler H: The regulatory $\mathrm{c} 1$ locus of Zea mays encodes a protein with homology to myb proto-oncogene products and with structural similarities to transcriptional activators. EMBO J 1987, 6(12):3553-3558.

7. Ng DW, Abeysinghe JK, Kamali M: Regulating the Regulators: The Control of Transcription Factors in Plant Defense Signaling. Int J Mol Sci 2018, 19(12).

8. Baldoni E, Genga A, Cominelli E: Plant MYB Transcription Factors: Their Role in Drought Response Mechanisms. Int J Mol Sci 2015, 16(7):15811-15851.

9. Li J, Han G, Sun C, Sui N: Research advances of MYB transcription factors in plant stress resistance and breeding. Plant Signal Behav 2019, 14(8):1613131.

10. Feller A, Machemer K, Braun EL, Grotewold E: Evolutionary and comparative analysis of MYB and bHLH plant transcription factors. Plant J 2011, 66(1):94-116.

11. Zhang S, Chen Y, He X, Du J, Zhang R, Ma Y, Hu X, Zhang Z, Chen Q, Wan X: Identification of MYB Transcription Factors Regulating Theanine Biosynthesis in Tea Plant Using Omics-Based Gene Coexpression Analysis. J Agric Food Chem 2020, 68(3):918-926.

12. Arce-Rodríguez ML, Ochoa-Alejo N: An R2R3-MYB Transcription Factor Regulates Capsaicinoid Biosynthesis. Plant Physiol 2017, 174(3):1359-1370.

13. Akagi T, Ikegami A, Tsujimoto T, Kobayashi S, Sato A, Kono A, Yonemori K: DkMyb4 is a Myb transcription factor involved in proanthocyanidin biosynthesis in persimmon fruit. Plant Physiol 2009, 151(4):2028-2045. 
14. Jian W, Cao H, Yuan S, Liu Y, Lu J, Lu W, Li N, Wang J, Zou J, Tang N et al: SIMYB75, an MYBtype transcription factor, promotes anthocyanin accumulation and enhances volatile aroma production in tomato fruits. Hortic Res 2019, 6:22.

15. Bac-Molenaar JA, Fradin EF, Rienstra JA, Vreugdenhil D, Keurentjes JJ: GWA Mapping of Anthocyanin Accumulation Reveals Balancing Selection of MYB90 in Arabidopsis thaliana. PLOS ONE 2015, 10(11):e0143212.

16. Muñoz-Gómez S, Suárez-Baron H, Alzate JF, González F, Pabón-Mora N: Evolution of the Subgroup 6 R2R3-MYB Genes and Their Contribution to Floral Color in the Perianth-Bearing Piperales. Frontiers in plant science 2021, 12:633227.

17. Huang D, Tang Z, Fu J, Yuan Y, Deng X, Xu Q: CsMYB3 and CsRuby1 form an 'Activator-andRepressor' Loop for the Regulation of Anthocyanin Biosynthesis in Citrus. Plant Cell Physio/ 2020, 61(2):318-330.

18. Ballester AR, Molthoff J, de Vos R, Hekkert B, Orzaez D, Fernández-Moreno JP, Tripodi P, Grandillo $\mathrm{S}$, Martin $\mathrm{C}$, Heldens $\mathrm{J}$ et al: Biochemical and molecular analysis of pink tomatoes: deregulated expression of the gene encoding transcription factor SIMYB12 leads to pink tomato fruit color. Plant Physiol 2010, 152(1):71-84.

19. Ito M, Araki S, Matsunaga S, Itoh T, Nishihama R, Machida Y, Doonan JH, Watanabe A: G2/Mphase-specific transcription during the plant cell cycle is mediated by c-Myb-like transcription factors. Plant Cell 2001, 13(8):1891-1905.

20. Araki S, Ito M, Soyano T, Nishihama R, Machida Y: Mitotic cyclins stimulate the activity of c-Myblike factors for transactivation of G2/M phase-specific genes in tobacco. J Biol Chem 2004, 279(31):32979-32988.

21. Sun X, Gong SY, Nie XY, Li Y, Li W, Huang GQ, Li XB: A R2R3-MYB transcription factor that is specifically expressed in cotton (Gossypium hirsutum) fibers affects secondary cell wall biosynthesis and deposition in transgenic Arabidopsis. Physiol Plant 2015, 154(3):420-432.

22. Kobayashi K, Suzuki T, Iwata E, Magyar Z, Bögre L, Ito M: MYB3Rs, plant homologs of Myb oncoproteins, control cell cycle-regulated transcription and form DREAM-like complexes.

Transcription 2015, 6(5):106-111.

23. Zhu X, Liang W, Cui X, Chen M, Yin C, Luo Z, Zhu J, Lucas WJ, Wang Z, Zhang D:

Brassinosteroids promote development of rice pollen grains and seeds by triggering expression of Carbon Starved Anther, a MYB domain protein. Plant J 2015, 82(4):570-581.

24. Chen T, Li W, Hu X, Guo J, Liu A, Zhang B: A Cotton MYB Transcription Factor, GbMYB5, is Positively Involved in Plant Adaptive Response to Drought Stress. Plant Cell Physio/ 2015, 56(5):917-929. 

to chilling stress in OsMYB3R-2 transgenic rice is mediated by alteration in cell cycle and ectopic expression of stress genes. Plant Physiol 2009, 150(1):244-256.

26. Wang Y, Li F, He Q, Bao Z, Zeng Z, An D, Zhang T, Yan L, Wang H, Zhu S et al: Genomic analyses provide comprehensive insights into the domestication of bast fiber crop ramie (Boehmeria nivea). Plant $J 2021$.

27. Mu L, Cai M, Wang Z, Liu J, Liu T, Wanapat M, Huang B: Assessment of ramie leaf (Boehmeria nivea L. gaud) as an animal feed supplement in P.R. China. Trop Anim Health Prod 2020, 52(1):115-121.

28. Kiprioti, M., Alexopoulou, E., Vafeiadakis, T., Kipriotis, Heping, Crops XJI, Products: Ramie and kenaf as feed crops. 2015.

29. Luan MB, Jian JB, Chen P, Chen JH, Chen JH, Gao Q, Gao G, Zhou JH, Chen KM, Guang XM et al: Draft genome sequence of ramie, Boehmeria nivea (L.) Gaudich. Mol Ecol Resour 2018, 18(3):639-645.

30. Jiang C, Gu X, Peterson T: Identification of conserved gene structures and carboxy-terminal motifs in the Myb gene family of Arabidopsis and Oryza sativa L. ssp. indica. Genome Biol 2004, 5(7):R46.

31. Liu C, Hao J, Qiu M, Pan J, He Y: Genome-wide identification and expression analysis of the MYB transcription factor in Japanese plum (Prunus salicina). Genomics 2020, 112(6):4875-4886.

32. Li Z, Peng R, Tian Y, Han H, Xu J, Yao Q: Genome-Wide Identification and Analysis of the MYB Transcription Factor Superfamily in Solanum lycopersicum. Plant Cell Physiol 2016, 57(8):1657-1677.

33. Williams CE, Grotewold E: Differences between plant and animal Myb domains are fundamental for DNA binding activity, and chimeric Myb domains have novel DNA binding specificities. $J$ Biol Chem 1997, 272(1):563-571.

34. Guo Y, Zhu C, Zhao S, Zhang S, Wang W, Fu H, Li X, Zhou C, Chen L, Lin Y et al: De novo transcriptome and phytochemical analyses reveal differentially expressed genes and characteristic secondary metabolites in the original oolong tea (Camellia sinensis) cultivar 'Tieguanyin' compared with cultivar 'Benshan'. BMC Genomics 2019, 20(1):265-276.

35. Du H, Yang SS, Liang Z, Feng BR, Liu L, Huang YB, Tang YX: Genome-wide analysis of the MYB transcription factor superfamily in soybean. BMC Plant Bio/ 2012, 12:106.

36. Schilling S, Kennedy A, Pan S, Jermiin LS, Melzer R: Genome-wide analysis of MIKC-type MADSbox genes in wheat: pervasive duplications, functional conservation and putative neofunctionalization. New Phytol 2020, 225(1):511-529. 
37. Holub EB: The arms race is ancient history in Arabidopsis, the wildflower. Nat Rev Genet 2001, 2(7):516-527.

38. Kranz HD, Denekamp M, Greco R, Jin H, Leyva A, Meissner RC, Petroni K, Urzainqui A, Bevan M, Martin $C$ et al: Towards functional characterisation of the members of the R2R3-MYB gene family from Arabidopsis thaliana. Plant $J$ 1998, 16(2):263-276.

39. Cannon SB, Mitra A, Baumgarten A, Young ND, May G: The roles of segmental and tandem gene duplication in the evolution of large gene families in Arabidopsis thaliana. BMC Plant Bio/ 2004, 4:10.

40. Lynch M, Conery JS: The evolutionary fate and consequences of duplicate genes. Science 2000, 290(5494):1151-1155.

41. Liu C, Zeng L, Zhu S, Wu L, Wang Y, Tang S, Wang H, Zheng X, Zhao J, Chen X et al: Draft genome analysis provides insights into the fiber yield, crude protein biosynthesis, and vegetative growth of domesticated ramie (Boehmeria nivea L. Gaud). DNA Res 2018, 25(2):173-181.

42. Huang KY, Zhu AG, Chen XR, Shi YL, Tang Q, Wang XF, Sun ZM, Luan MB, Chen JH: Comparative transcriptomics reveals the selection patterns of domesticated ramie. Ecol Evol 2019, 9(12):7057-7068.

43. Liu C, Xie T, Chen C, Luan A, Long J, Li C, Ding Y, He Y: Genome-wide organization and expression profiling of the R2R3-MYB transcription factor family in pineapple (Ananas comosus). $B M C$ Genomics 2017, 18(1):503.

44. Ming R, VanBuren R, Wai CM, Tang H, Schatz MC, Bowers JE, Lyons E, Wang ML, Chen J, Biggers $\mathrm{E}$ et al: The pineapple genome and the evolution of CAM photosynthesis. Nat Genet 2015, 47(12):14351442.

45. Stracke R, Werber M, Weisshaar B: The R2R3-MYB gene family in Arabidopsis thaliana. Curr Opin Plant Biol 2001, 4(5):447-456.

46. Thiel T, Graner A, Waugh R, Grosse I, Close TJ, Stein N: Evidence and evolutionary analysis of ancient whole-genome duplication in barley predating the divergence from rice. BMC Evol Bio/ 2009, 9:209.

47. Si W, Hang T, Guo M, Chen Z, Liang Q, Gu L, Ding T: Whole-Genome and Transposed Duplication Contributes to the Expansion and Diversification of TLC Genes in Maize. Int J Mol Sci 2019, 20(21).

48. Chen Q, Liu Y, Maere S, Lee E, Van Isterdael G, Xie Z, Xuan W, Lucas J, Vassileva V, Kitakura S et al: A coherent transcriptional feed-forward motif model for mediating auxin-sensitive PIN3 expression during lateral root development. Nat Commun 2015, 6:8821.

49. Pierrugues O, Brutesco C, Oshiro J, Gouy M, Deveaux Y, Carman GM, Thuriaux P, Kazmaier M: Lipid phosphate phosphatases in Arabidopsis. Regulation of the AtLPP1 gene in response to stress. $J$ 
Biol Chem 2001, 276(23):20300-20308.

50. Yamaguchi-Shinozaki K, Shinozaki K: Transcriptional regulatory networks in cellular responses and tolerance to dehydration and cold stresses. Annu Rev Plant Biol 2006, 57:781-803.

51. Ashburner M, Ball CA, Blake JA, Botstein D, Butler H, Cherry JM, Davis AP, Dolinski K, Dwight SS, Eppig JT et al: Gene ontology: tool for the unification of biology. The Gene Ontology Consortium. Nat Genet 2000, 25(1):25-29.

52. Chen S, Niu X, Guan Y, Li H: Genome-Wide Analysis and Expression Profiles of the MYB Genes in Brachypodium distachyon. Plant Cell Physiol 2017, 58(10):1777-1788.

53. Smita S, Katiyar A, Chinnusamy V, Pandey DM, Bansal KC: Transcriptional Regulatory Network Analysis of MYB Transcription Factor Family Genes in Rice. Frontiers in plant science 2015, 6:1157.

54. Katiyar A, Smita S, Lenka SK, Rajwanshi R, Chinnusamy V, Bansal KC: Genome-wide classification and expression analysis of MYB transcription factor families in rice and Arabidopsis. $B M C$ Genomics 2012, 13:544.

55. Chen X, Wang P, Gu M, Lin X, Hou B, Zheng Y, Sun Y, Jin S, Ye N: R2R3-MYB transcription factor family in tea plant (Camellia sinensis): Genome-wide characterization, phylogeny, chromosome location, structure and expression patterns. Genomics 2021, 113(3):1565-1578.

56. Feng X, Gao G, Yu C, Zhu A, Chen J, Chen K, Wang X, Abubakar AS, Chen P: Transcriptome and metabolome analysis reveals anthocyanin biosynthesis pathway associated with ramie (Boehmeria nivea (L.) Gaud.) leaf color formation. BMC Genomics 2021, 22(1):684.

57. Lloyd A, Brockman A, Aguirre L, Campbell A, Bean A, Cantero A, Gonzalez A: Advances in the MYB-bHLH-WD Repeat (MBW) Pigment Regulatory Model: Addition of a WRKY Factor and Co-option of an Anthocyanin MYB for Betalain Regulation. Plant Cell Physiol 2017, 58(9):1431-1441.

58. Yoshida K, Ma D, Constabel CP: The MYB182 protein down-regulates proanthocyanidin and anthocyanin biosynthesis in poplar by repressing both structural and regulatory flavonoid genes. Plant Physio/ 2015, 167(3):693-710.

59. Cavallini E, Matus JT, Finezzo L, Zenoni S, Loyola R, Guzzo F, Schlechter R, Ageorges A, ArceJohnson $\mathrm{P}$, Tornielli GB: The phenylpropanoid pathway is controlled at different branches by a set of R2R3-MYB C2 repressors in grapevine. Plant Physiol 2015, 167(4):1448-1470.

60. Zimmermann IM, Heim MA, Weisshaar B, Uhrig JF: Comprehensive identification of Arabidopsis thaliana MYB transcription factors interacting with R/B-like BHLH proteins. Plant J 2004, 40(1):22-34.

61. Zhou B, Leng J, Ma Y, Fan P, Li Y, Yan H, Xu Q: BrmiR828 targets BrPAP1, BrMYB82, and BrTAS4 involved in the light induced anthocyanin biosynthetic pathway in Brassica rapa. Int J Mol Sci 2020, 
62. She W, Zhu S, Jie Y, Xing H, Cui G: Expression profiling of cadmium response genes in ramie (Boehmeria nivea L.) root. Bull Environ Contam Toxicol 2015, 94(4):453-459.

63. Agarwal P, Mitra M, Banerjee S, Roy S: MYB4 transcription factor, a member of R2R3-subfamily of MYB domain protein, regulates cadmium tolerance via enhanced protection against oxidative damage and increases expression of PCS1 and MT1C in Arabidopsis. Plant Sci 2020, 297:110501.

64. Fichman Y, Zandalinas SI, Sengupta S, Burks D, Myers RJ, Jr., Azad RK, Mittler R: MYB30 Orchestrates Systemic Reactive Oxygen Signaling and Plant Acclimation. Plant Physio/ 2020, 184(2):666675.

65. Zhang P, Wang R, Ju Q, Li W, Tran LP, Xu J: The R2R3-MYB Transcription Factor MYB49 Regulates Cadmium Accumulation. Plant Physiol 2019, 180(1):529-542.

66. Seo PJ, Lee SB, Suh MC, Park MJ, Go YS, Park CM: The MYB96 transcription factor regulates cuticular wax biosynthesis under drought conditions in Arabidopsis. Plant Cel/ 2011, 23(3):1138-1152.

67. Mehrtens F, Kranz H, Bednarek P, Weisshaar B: The Arabidopsis transcription factor MYB12 is a flavonol-specific regulator of phenylpropanoid biosynthesis. Plant Physiol 2005, 138(2):1083-1096.

68. Baudry A, Heim MA, Dubreucq B, Caboche M, Weisshaar B, Lepiniec L: TT2, TT8, and TTG1 synergistically specify the expression of BANYULS and proanthocyanidin biosynthesis in Arabidopsis thaliana. Plant J 2004, 39(3):366-380.

69. Zhao J, Zhang W, Zhao Y, Gong X, Guo L, Zhu G, Wang X, Gong Z, Schumaker KS, Guo Y: SAD2, an importin -like protein, is required for UV-B response in Arabidopsis by mediating MYB4 nuclear trafficking. Plant Cell 2007, 19(11):3805-3818.

70. Smirnova E, Marquis V, Poirier L, Aubert Y, Zumsteg J, Ménard R, Miesch L, Heitz T: Jasmonic Acid Oxidase 2 Hydroxylates Jasmonic Acid and Represses Basal Defense and Resistance Responses against Botrytis cinerea Infection. Mol Plant 2017, 10(9):1159-1173.

71. Miura K, Renhu N, Suzaki T: The PHD finger of Arabidopsis SIZ1 recognizes trimethylated histone H3K4 mediating SIZ1 function and abiotic stress response. Communications biology 2020, 3(1):23.

72. Ma Q, Xia Z, Cai Z, Li L, Cheng Y, Liu J, Nian H: GmWRKY16 Enhances Drought and Salt Tolerance Through an ABA-Mediated Pathway in Arabidopsis thaliana. Frontiers in plant science 2018, 9:1979.

73. Kim D, Ntui VO, Xiong L: Arabidopsis YAK1 regulates abscisic acid response and drought resistance. FEBS Lett 2016, 590(14):2201-2209. 
74. Wilkins MR, Gasteiger E, Bairoch A, Sanchez JC, Williams KL, Appel RD, Hochstrasser DF: Protein identification and analysis tools in the ExPASy server. Methods Mol Biol 1999, 112:531-552.

75. Chen C, Chen H, Zhang Y, Thomas HR, Frank MH, He Y, Xia R: TBtools: An Integrative Toolkit Developed for Interactive Analyses of Big Biological Data. Mol Plant 2020, 13(8):1194-1202.

76. Bailey TL, Boden M, Buske FA, Frith M, Grant CE, Clementi L, Ren J, Li WW, Noble WS: MEME SUITE: tools for motif discovery and searching. Nucleic Acids Res 2009, 37(Web Server issue):W202-208.

77. Krzywinski M, Schein J, Birol I, Connors J, Gascoyne R, Horsman D, Jones SJ, Marra MA: Circos: an information aesthetic for comparative genomics. Genome Res 2009, 19(9):1639-1645.

78. Wang Y, Tang H, Debarry JD, Tan X, Li J, Wang X, Lee TH, Jin H, Marler B, Guo H et al: MCScanX: a toolkit for detection and evolutionary analysis of gene synteny and collinearity. Nucleic Acids Res 2012, 40(7):e49.

79. Wang D, Zhang Y, Zhang Z, Zhu J, Yu J: KaKs_Calculator 2.0: a toolkit incorporating gammaseries methods and sliding window strategies. Genomics, proteomics \& bioinformatics 2010, 8(1):77-80.

80. Kumar S, Stecher G, Tamura K: MEGA7: Molecular Evolutionary Genetics Analysis Version 7.0 for Bigger Datasets. Mol Biol Evol 2016, 33(7):1870-1874.

81. Du H, Feng BR, Yang SS, Huang YB, Tang YX: The R2R3-MYB transcription factor gene family in maize. PLOS ONE 2012, 7(6):e37463.

82. Wang H, Sun S, Zhou Z, Qiu Z, Cui X: Rapid analysis of anthocyanin and its structural modifications in fresh tomato fruit. Food Chem 2020, 333:127439.

83. Gao G, Xiong H, Chen J, Chen K, Chen P, Yu C, Zhu A: Hydroponic method for ramie and removal of nitrogen and phosphorus from livestock wastewater. Int J Phytoremediation 2018, 20(6):545-551.

84. Xu L, Dong Z, Fang L, Luo Y, Wei Z, Guo H, Zhang G, Gu YQ, Coleman-Derr D, Xia Q et al: OrthoVenn2: a web server for whole-genome comparison and annotation of orthologous clusters across multiple species. Nucleic Acids Res 2019, 47(W1):W52-w58.

85. Szklarczyk D, Morris JH, Cook H, Kuhn M, Wyder S, Simonovic M, Santos A, Doncheva NT, Roth A, Bork $P$ et al: The STRING database in 2017: quality-controlled protein-protein association networks, made broadly accessible. Nucleic Acids Res 2017, 45(D1):D362-d368.

\section{Figures}

Figure 1 
Consensus sequence and the level of conservation of R2R3-MYB domains from ramie. The sequence logos of the R2 and R3 MYB repeats are based on full-length alignments of all ramie R2R3-MYB domains. The bit score exhibits the information content for each position in the sequence. The position of the three a-helices were marked (Helix 1 to 3 ). The conserved tryptophan residues (Trp, W) in the MYB domain are marked with black asterisks.

\section{Figure 2}

Phylogenetic relationships, gene structure and architecture of conserved protein motifs in BnGR2R3-MYB genes. A. The maximum likelihood phylogenetic includes 105 R2R3-MYB proteins from ramie and 23 representatives from Arabidopsis B. The motif architecture of ramie MYB proteins. 20 different motifs are displayed in different colored boxes. C. Exon-intron structure of ramie MYB genes. Green boxes indicate exons; blue boxes indicate untranslated 5'- and 3'- regions; black lines indicate introns. R2, R3 MYB domain are highlighted by boxes of different shape and color. 


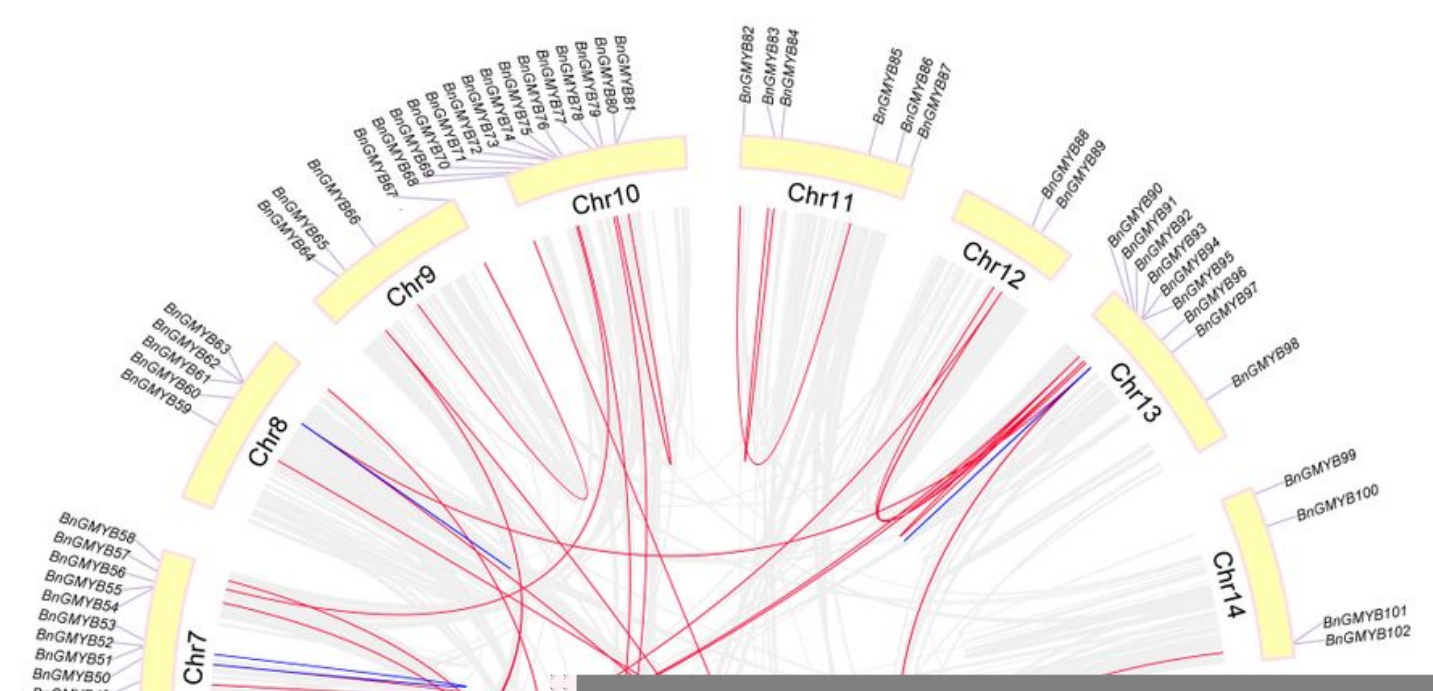

\section{Figure 3}

Schematic representations for the chromosomal distribution and inter-chromosomal relationships of ramie R2R3-MYB genes. Gray lines indicate all synteny blocks in the ramie genome, and the red lines indicate duplicated MYB gene pairs. Gene and chromosome names are displayed on the tip and inside of the chromosome, respectively. 


\section{Figure 4}

Synteny analyses of MYB genes between ramie and five representative plants: $B$. nivea, $C$. sativa, $A$. venetum, $O$. sativa, $Z$. mays and $A$. thaliana. Gray lines in the background indicate the collinear blocks within ramie and other plant genomes, while the red lines highlight the syntenic MYB gene pairs.

\section{Figure 5}

Neighbor-joining [34] tree showing relationships among R2R3-MYB proteins from B. nivea [48], $A$. thaliana (At), O. sativa (Os), Z. mays [49], S. lycopersicum (SI) and A. comosus (Ac). MYB proteins from the six species were designated as $\mathrm{C} 1$ to $\mathrm{C} 35$. The table on the right indicated the number of the subgroup members and function of clades in each species.

\section{Figure 6}

Analysis of cis-elements from promoter region of the BnGR2R3-MYB genes. The figure on the left shows the distribution of four cis-acting elements upstream promoter regions $(1000 \mathrm{bp})$. On the right is the heat map of the quantity of plant growth, stress responsive and phytohormone responsive cis-elements, represented in brown, blue and green, respectively, circle sizes indicate the number of cis-elements.

\section{Figure 7}

BnGMYB Gene Ontology (GO) functional classification. (A) BnGMYB TFs were divided into categories with three independent ontologies. (B) The Top 20 of GO enrichment in biological processes.

\section{Figure 8}

Hierarchical clustering of expression profiles of ramie R2R3-MYB genes in 9 samples including different tissues and developmental stages. Log2(FPKM +1 ) values were displayed according to the color code. Detailed FPKM values were listed in Table S7.

\section{Figure 9}

UPLC-QTOF-MS method for the detection of anthocyanin in HX_1 and ZZ_1. 


\section{Figure 10}

Phylogenetic tree and gene expression analysis of R2R3-MYB transcription factors associated with anthocyanin synthesis.

\section{Figure 11}

The relative expression levels of selected MYB genes in two ramie varieties in different tissue sites (root, stem, leaf) at different periods under $\mathrm{Cd}^{+2}$ treatment ( $\left.0 \mathrm{~h}, 6 \mathrm{~h}, 12 \mathrm{~h}, 24 \mathrm{~h}, 48 \mathrm{~h}\right)$. A represents root; $\mathrm{B}$ represents stem; $C$ represents leaf. The error bar represents the standard deviation of the three biological duplicates. * represents significant difference $(P<0.05)$, ** represents extremely significant difference $(P<$ $0.01)$.

\section{Figure 12}

The protein-protein interaction network for BnGR2R3-MYB proteins based on their Arabidopsis orthologs. The red solid circles indicated cadmium stress-related MYB transcription factors, the olive squares represented stress-response proteins, and the green squares were flavonoid synthesis related proteins.

\section{Supplementary Files}

This is a list of supplementary files associated with this preprint. Click to download.

- Additionalfile1.xlsx

- Additionalfile2.txt

- Additionalfile3.xlsx

- Additionalfile4.xlsx

- Additionalfile5.xlsx

- Additionalfile6.xlsx

- Additionalfile7.xlsx

- Additionalfile8.xlsx

- Additionalfile9.docx

- Additionalfile10.pdf 
- Additionalfile11.pdf

Page 29/29 